\title{
Uma análise multicritério dos indicadores de liquidez e rentabilidade de empresas Brasileiras da construção civil
}

Alessandra dos Santos Simão alessandra_simao@id.uff.br Universidade Federal Fluminense (UFF), Niterói, Rio de Janeiro, Brasil

Luciane Ferreira Alcoforado lucianealcoforado@gmail.com Universidade Federal Fluminense (UFF), Niterói, Rio de Janeiro, Brasil

\section{Orlando Celso Longo} orlandolongo@gmail.com Universidade Federal Fluminense (UFF) Niterói, Rio de Janeiro, Brasil

\section{Danillo Araújo dos Santos} enguva.danillo@gmail.com Universidade Federal Fluminense (UFF) Niterói, Rio de Janeiro, Brasil

\section{Fábio dos Santos}

santfab@gmail.com

Universidade Federal Fluminense (UFF)

Niterói, Rio de Janeiro, Brasil

\author{
RESUMO
}

A pesquisa objetiva definir o ranking das empresas do setor de construção civil listadas na BM\&FBovespa baseado nos indicadores de liquidez e rentabilidade com o método Analytic Hierarchy Process, AHP Multiplicativo. Para a análise foram coletados os dados das demonstrações financeiras: Balanço Patrimonial e Demonstração do Resultado do Exercício (DRE), extraídos do site da Comissão de Valores Mobiliários (CVM) e analisados usando-se o Método AHP Multiplicativo para estabelecer os rankings anuais e gerais. Constatou-se que nos indicadores de liquidez, destacam-se as empresas: Adolpho Lindenberg (em 2010, 2012 e 2013), Rossi (2014 e 2015) e Even (em 2011). Já nos indicadores de rentabilidade, se destacam as empresas Rossi (em 2010, 2011 e 2013), Brookfield (em 2012), Tecnisa (em 2014) e Rodobens (em 2015). Com os resultados, elaborou-se um ranking geral em relação ao período analisado, destacando-se em primeiro lugar a Adolpho Lindenberg e em segundo a empresa Rossi. Os resultados corroboram com Assaf Neto e Lima (2014), que esclarece que a empresa pode possuir boa capacidade de liquidar suas obrigações, contudo não necessariamente apresentará o mesmo potencial de rentabilidade, ou seja, a empresa pode não usufruir de bom resultado econômico.

PALAVRAS-CHAVE: Análise de indicadores. Liquidez. Rentabilidade. AHP Multiplicativo. 


\section{INTRODUÇÃO}

Existem várias maneiras de avaliar o desempenho de uma empresa, uma das formas mais comuns é por meio da análise de seus demonstrativos financeiros. $O$ uso de indicadores para análise de empresas é uma ferramenta bastante útil, tanto que ludícibus (2017) afirma que a técnica de análise financeira por quocientes é um dos mais importantes desenvolvimentos da contabilidade.

A análise econômico-financeira tem destaque em pesquisas anteriores, pois é um instrumento que permite a tomada de decisão sobre investimentos, operações e financiamentos. Ainda permite realizar um estudo para compreensão das variações ocorridas no patrimônio, endividamento, lucratividade, rentabilidade e principalmente o posicionamento da empresa perante as demais do setor, além de ser uma excelente ferramenta para análise dos pontos que necessitam de atenção para a melhoria do desempenho da empresa (GITMAN, 2010).

É importante entender as demonstrações contábeis, também denominadas de demonstrações financeiras, para administrar bem o negócio em que a empresa está inserida. Estas representam um canal de comunicação da empresa com os diversos usuários internos e externos. Elas permitem uma rápida visão da situação da empresa, um ponto de partida para análises posteriores e servem de base para planejamento e elaboração de orçamentos (GITMAN, 2010).

A construção de um ranking, baseado em informações que permitam obter o desempenho econômico-financeiro, é de grande valia para os investidores, visto que os rankings pesquisados disponíveis em sites e revistas especializadas em negócios e construção civil não consideram os índices, tais como: Revista Pini Construção e Mercado, que considera critérios técnicos e comerciais; Revista $O$ Empreiteiro, que considera a variação percentual de receita bruta das empresas em relação a contratos públicos e privados; o site ITC - Inteligência Empresarial da Construção, que considera o número de metros quadrados construídos e número de obras concluídas por ano. Já CBIC - Câmara Brasileira da Indústria da Construção e a Revista Exame apenas divulgam o ranking construído pela ITC.

Para estabelecer um ranking, pode-se atribuir pesos, isto é, valores que assinalam a importância de cada elemento dentro do conjunto analisado. Esta classificação pode ser realizada por meio da aplicação do Analytic Hierarchy Process (AHP), desenvolvido por Thomas L. Saaty. O modelo estabelecido pelo método de análise hierárquica, de acordo com Saaty (1991, p. 1), "têm de incluir e medir todos os fatores importantes, qualitativa e quantitativamente mensuráveis, sejam eles tangíveis ou intangíveis".

A utilização dos Métodos Multicritérios pode auxiliar de maneira eficiente a análise dos indicadores contábil-financeiros. De acordo com Gomes, Araya e Carignano (2004), o Apoio Multicritério à Decisão (AMD) consiste em um conjunto de métodos e técnicas para auxiliar ou apoiar a tomada de decisões, quando da presença de uma multiplicidade de critérios.

O AHP é um dos métodos multicritérios de apoio a decisão mais utilizados (HERNANDÉZ; MARINS; CASTRO, 2012), apresentando o principal benefício onde, os valores dos julgamentos das comparações paritárias são baseados em experiência, intuição e em dados físicos, podendo lidar com aspectos qualitativos e quantitativos de um problema de decisão (MENEZES, 2015). O método objetiva 
tornar o processo mais neutro, objetivo, válido e o mais transparente possível (SHIMIZU, 2006).

Diante do exposto, esta pesquisa busca responder a seguinte questão de pesquisa: Quais são os rankings em relação aos indicadores de liquidez e rentabilidade das empresas do setor de construção civil listadas na Bovespa? Para tanto, a pesquisa objetiva em definir por meio dos indicadores de liquidez e rentabilidade, uma hierarquização do setor construção civil de empresas listadas na BM\&FBovespa utilizando o método AHP Multiplicativo (Análise Hierárquica de Processo - Analytic Hierarchy Process).

Diante da globalização econômica, o perfil de atividade das empresas foi alterado, o que levou a estas se adaptarem a uma nova realidade em que os mercados se tornaram maiores e mais competitivos. Dessa forma, veio a maior necessidade de compatibilizar um sistema de avaliação de desempenho dentro das organizações. Neste sentido, o método AHP possibilita a hierarquização dos indicadores de maneira que é possível verificar a ordem de importância dos mesmos no momento da análise, ou seja, quais os índices que melhor identificam a situação de uma empresa.

A partir desta introdução, este estudo está estruturado da seguinte forma: será apresentada uma breve discussão acerca dos métodos de tomada de decisão com múltiplos critérios, o método AHP multiplicativo e análise multicritério para indicadores; na sequência serão apresentados os procedimentos metodológicos seguidos em sua elaboração e, posteriormente serão apresentados a análise e discussão dos resultados. Por fim, serão apresentadas as considerações finais.

\section{MÉTODOS DE TOMADA DE DECISÃO COM MÚLTIPLOS CRITÉRIOS}

No processo decisório, Saaty (1991) esclarece a necessidade de avaliação da alternativa que melhor satisfaça o conjunto de critérios pretendidos. Ainda, adverte que "a melhor solução para um problema multicritério não é aquela obtida por um método matemático complexo, mas aquela preferida, aceita, entendida e defendida pelo decisor".

O processo decisório compreende a escolha da melhor decisão ponderando múltiplos critérios, alternativas ou múltiplos objetivos. Os Métodos de Programação Matemática podem ser inadequados para tratar variáveis qualitativas, ou não vai possibilitar que se encontre uma solução que vai ser melhor do que as outras para todos os critérios (SHIMIZU, 2006).

Primeiramente, ocorre uma divergência na literatura sobre a denominação destes métodos, Tomada de Decisão com Múltiplos Critérios (MCDM - Multiple Criteria Decision Making), Auxílio à Decisão com Múltiplos Critérios (MCDA Multiple Criteria Decision Aid) e ainda, alguns autores preferem AMD - Apoio Multicritério à Decisão. Neste trabalho, adota-se a denominação de AMD sem distinção dos critérios usados para a sua classificação.

O AMD trata de um estudo da inclusão de critérios conflitantes na tomada de decisão. O método objetiva tornar o processo mais neutro, objetivo, válido e o mais transparente possível (SHIMIZU, 2006). 
O AMD é uma das metodologias de decisão mais utilizadas, pois possibilita avaliar ao mesmo tempo as soluções que atendem a mais de um objetivo, por mais de um critério, podendo ou não abordar interdependências entre essas soluções (alternativas) consideradas como elementos de avaliação proporcionando melhor qualidade, racionalidade e eficiência ao processo de tomada de decisão.

A contar da década de 1970, advém uma nova etapa no processo decisório, isto devido à insuficiência dos recursos financeiros e humanos, e o aumento no custo destes recursos fizeram com que os interesses se voltassem para os domínios dos múltiplos critérios.

Com este novo cenário, surgiram diferentes métodos que fornecem uma ordenação das alternativas com base em determinados princípios e outros que, além desta ordenação, oferecem uma medida do desempenho das alternativas, considerando todos os critérios (desempenho global).

Em ambientes de tomada de decisão complexa, a mesma envolve itens tangíveis e intangíveis com critérios e múltiplas alternativas. Para essa problemática, em que envolve fatores quanti e qualitativo, alguns especialistas desenvolveram vários múltiplos métodos a tomada de decisões (ELECTRE, Macbeth, SMART PROMETHEE, AHP, ANP, etc). Todos são baseados em quatro etapas: modelagem pesos de avaliação problema, pesos de agregação e análise sensibilidade (ALTAMIRANO CORRO et al., 2015)

Cada método envolve um distinto processo de modelagem, em que uma representação simplificada da realidade, procura identificar e destacar os elementos mais importantes à tomada de decisão. Contudo, o processo decisório envolve, quase sempre, a escolha da melhor decisão considerando múltiplos critérios, fatores ou objetivos. E neste contexto, verifica-se conforme Shimizu (2006) a necessidade em esclarecer cada termo, pois nos trabalhos de alguns autores os termos critério, objetivo e restrição são usados como se fossem sinônimos.

- Critérios: são os fatores ou variáveis quantitativas ou qualitativas consideradas na escolha da melhor alternativa de decisão;

- Função objetivo: é uma função matemática que representa um ou mais objetivos a serem otimizados; e

- Restrições: são as condições ou limites impostos aos critérios ou fatores para definir o espaço das soluções possíveis ou viáveis de um problema de otimização.

Os critérios são os objetivos e restrições "relaxadas" utilizadas nos métodos de decisão com múltiplos critérios, como no método AHP em que este trabalho aborda.

\section{O MÉTODO AHP MULTIPLICATIVO}

O método Análise Hierárquica de Processo (Analytic Hierarchy Process, AHP), permite realizar uma análise multicritério que ajuda no processo decisório (MATTANA et al., 2012). O método desenvolvido por Saaty na década de 70 utilizou a comparação pareada para calcular os pesos dos elementos em cada 
nível e determina o desempenho global (peso final) levando em consideração todos os critérios envolvidos (SAATY, 2013).

Para Mattana et al. (2012) um dos objetivos da AHP é aumentar a objetividade e diminuir a subjetividade da decisão, considerando a subjetividade de alguns parâmetros com a construção de hierarquias e atribuição de pesos de acordo com o grau de importância.

De acordo com Pereira et al. (2012) o método é desenvolvido em três princípios básicos:

- Construção hierárquica, pela qual são identificados os elementos-chave para a tomada de decisão;

- Definição de prioridades;

- Consistência lógica do modelo de priorização construído.

No Método AHP Clássico, o agente externo sua preferência entre duas ou mais alternativas comparando-as de acordo com a escala fundamental (SAATY, 2008). Este procedimento pode gerar a ocorrência da inversão de ordem no emprego do método AHP Clássico, caracterizada pela possibilidade da alteração da ordem das prioridades globais das alternativas devido à remoção de uma alternativa ou introdução de uma nova alternativa ao problema, e evidencia que na fase de modelagem pode ter ocorrido falha (LOOTSMA, 1993).

Com a finalidade de evitar a ocorrência deste problema, foram desenvolvidas três versões para o Método AHP Clássico: i) o Método AHP Referenciado, de Watson \& Freeling em 1982; ii) o AHP B-G, de Belton \& Gear em 1985; e iii) e AHP Multiplicativo de Lootsma em 1993, que utiliza uma escala geométrica ao contrário da escala linear proposta por Saaty.

Lootsma propôs o Método AHP Multiplicativo para superar os seguintes pontos críticos identificados no método AHP Clássico de Saaty: i) a escala fundamental proposta por Saaty, em 1991, para quantificar os juízos humanos (a restrição de comparações pareadas sobre uma escala de 1 a 9 conduz ao agente de decisão em cometer inconsistências, ocorrendo limitação na flexibilidade na obtenção de comparações); ii) o uso do autovetor para calcular a prioridade das alternativas; e iii) as prioridades globais calculados por uma regra de média aritmética de agregação (LOOTSMA, 1993).

Lootsma (1993) ainda defende que a escala natural é mais ampla que a escala proposta por Saaty (escala fundamental), pois esta última não é uma escala geométrica e, portanto, os valores recíprocos propostos por Saaty poderiam produzir uma inconsistência que não está presente na mente do decisor. De acordo com o Método Multiplicativo, a comparação pareada das alternativas necessita ser fundamentada numa regra geométrica, e não aritmética, como ocorre no Método AHP Clássico. Assim, a Escala Natural é mais consistente que a Escala Fundamental, porque não permite a ocorrência dos desvios mencionados. Por fim, a Escala Natural possui uma natureza multiplicativa, proporcionando uma tendência natural para evitar a inversão de ordem.

O Método AHP Multiplicativo apresenta um funcionamento exatamente igual ao Método AHP Clássico, apresentando a diferença na escala de interpretação dos julgamentos. Enquanto que o AHP Clássico trabalha com uma 
escala linear, ou seja, a diferença entre os intervalos de julgamento permanece constante em toda a escala, o método AHP Multiplicativo utiliza-se de uma escala logarítmica na qual a diferença entre os intervalos de julgamentos aumenta à medida que os valores da escala aumentam.

O AHP Multiplicativo apresenta uma escala considerando preferências negativas (LOOTSMA, 1993). Estas escalas são utilizadas para quantificar as preferências de modo que se consiga estabelecer um ranking.

Quadro 1 - Escala natural de Lootsma

\begin{tabular}{|c|c|}
\hline Valor índice $\boldsymbol{\delta}$ ij & Interpretação \\
\hline-8 & Si é amplamente menos desejável que $\mathrm{Sj}$ \\
\hline-6 & Si é muito menos desejável que $\mathrm{Sj}$ \\
\hline-4 & Si é menos desejável que $\mathrm{Sj}$ \\
\hline-2 & Si é pouco menos desejável que $\mathrm{Sj}$ \\
\hline 0 & Si é indiferente a $\mathrm{Sj}$ \\
\hline 2 & Si é pouco mais desejável que $\mathrm{Sj}$ \\
\hline 4 & Si é mais desejável que $\mathrm{Sj}$ \\
\hline 6 & Si é muito mais desejável que $\mathrm{Sj}$ \\
\hline 8 & Si é amplamente mais desejável que $\mathrm{Sj}$ \\
\hline
\end{tabular}

Fonte: Lootsma (1993, p. 11)

Esta escala amplia a escala fundamental de Saaty, pois considera preferências negativas. Para determinar as matrizes de preferência, consideramse os índices $\delta$ ij do Quadro 1 para obter os valores dos $\gamma_{i j}$, ou seja, a matriz normalizada, utilizando a fórmula (GOMES; ARAYA; CARIGNANO, 2004, p. 64):

$$
\gamma_{i j}=e^{\gamma \delta_{i j}}
$$

Onde $\checkmark$ representa o fator de escala da escala geométrica utilizada para indicar os fatores de progressão para determinado número de categorias.

\section{PROCEDIMENTOS METODOLÓGICOS}

Para o desenvolvimento deste trabalho, foram realizadas 6 etapas:

- 1a fase: pesquisa bibliográfica acerca do tema; Consulta de artigos científicos sobre Análise Multicritério, Análise Hierárquica de Processo (AHP) e AHP Multiplicativo, na base de dados do Portal de Periódicos CAPES com o objetivo de consultar os artigos dos autores que desenvolveram os métodos apresentados no trabalho, realizada no período entre março de 2016 a junho de 2016.

- 2a fase: seleção de 12 empresas do setor de Construção Civil listadas no BM\&FBOVESPA, utilizando como fonte o ranking da ITC (Inteligência Empresarial da Construção) dos anos 2014 e 2015, conforme o Quadro 2;

O critério de seleção das empresas baseou-se em dois pontos: a empresa deveria constar no ranking da ITC nos anos de 2014 e 2015, e ainda estar listada no BM\&FBOVESPA. 
A escolha do período de análise dos rankings (anos 2014 e 2015) deve-se ao fato que até a seleção das empresas (fevereiro de 2016) o ranking ITC para o ano de 2016 ainda não tinha sido divulgado.

Quadro 2 - Empresas participantes da pesquisa

\begin{tabular}{|c|c|}
\hline Cód & Empresa \\
\hline E1 & Brookfield Incorporações \\
\hline E2 & Construtora Adolpho Lindenberg S.A. \\
\hline E3 & CR2 Empreendimentos Imobiliários S.A. \\
\hline E4 & Cyrela Brazil Realty S.A. \\
\hline E5 & Direcional Engenharia \\
\hline E6 & Even Construtora e Incorporadora S.A. \\
\hline E7 & Ez Tec Empreendimentos e Participações S.A \\
\hline E8 & Gafisa S.A. \\
\hline E9 & MRV Engenharia e Participações S.A. \\
\hline E10 & Rodobens Negócios Imobiliários S.A. \\
\hline E11 & Rossi Residencial S.A \\
\hline E12 & Tecnisa S.A. \\
\hline
\end{tabular}

Fonte: BM\&FBOVESPA (2016)

- 3a fase: Pesquisa documental com a realização de consulta em sites das empresas selecionadas do setor de Construção Civil, no site do BM\&FBOVESPA, e no site da CVM1 (Comissão de Valores Mobiliários) para selecionar os relatórios financeiros compreendidos em: Balanço Patrimonial (BP) e Demonstração do Resultado do Exercício (DRE), publicados no período de 2010 a 2015;

O critério de seleção do período de análise dos demonstrativos (de 2010 a 2015) baseou-se em dois pontos: 10 o processo de internacionalização da contabilidade iniciou-se em 2007, com a edição da Lei 11.638/07, entretanto somente a partir de 2010 que muitas empresas brasileiras foram obrigadas, inclusive as da construção civil, a adoção das IFRS (International Financial Reporting Standards) na elaboração e apresentação das suas demonstrações contábeis consolidadas, por reivindicação de órgãos como CVM (Comissão de Valores Mobiliários), CFC (Conselho Federal de Contabilidade), IBRACON (Instituto dos Auditores Independentes do Brasil) entre outros.

Assim, demonstrativos anteriores ao ano de 2010 não estavam padronizados a nova exigência dificultado a comparação dos demonstrativos.

O 2o ponto é que até o fechamento da seleção dos demonstrativos, não havia sido divulgado pelas empresas os relatórios do ano de 2016.

- 4a fase: aplicação presencial de pesquisa com 10 especialistas: engenheiros do setor de gestão de construção civil e professores de programas de pósgraduação da área contábil-financeiro e engenharia civil de uma Instituição de Ensino Superior Federal.

A pesquisa solicitava que os especialistas atribuíssem notas de 1 a 5 de acordo com o grau de importância dada aos indicadores na avaliação do

${ }^{1}$ CVM (Comissão de Valores Mobiliários) - Órgão oficial e governamental vinculada ao Ministério da Fazenda, com a finalidade de disciplinar e fiscalizar o mercado de valores mobiliários. Endereço eletrônico: www.cvm.gov.br 
desempenho econômico-financeiro (indicadores de: Liquidez, Rentabilidade, Atividade, Valor de Mercado e Estrutura de Capital), conforme Apêndice.

Os especialistas foram selecionados pela experiência, grau de conhecimento acerca do tema e a disponibilidade em participar da pesquisa;

- 5a fase: análise das demonstrações financeiras (BP e DRE) das empresas selecionadas, para extrair os indicadores de mensuração do risco (indicadores de liquidez), mensuração da rentabilidade (indicadores de rentabilidade), nos quais foram considerados, conforme a importância atribuída pelos especialistas: i) Liquidez: Liquidez Geral, Liquidez Corrente e Liquidez Seca; e (ii) Rentabilidade: Margem de Lucro Bruto, Margem de Lucro Líquido, Retorno sobre o Ativo (ROA) e Retorno sobre o Patrimônio Líquido (ROE); e

- 6a fase: Primeiramente, com os indicadores calculados realizou-se a análise de componentes principais para obtenção dos scores de cada grupo de indicadores com o auxílio do SPSS versão 15; por meio de planilha eletrônica realizou-se a comparação e análise dos indicadores previamente obtidos de acordo com o Método AHP Multiplicativo para hierarquização das empresas analisadas.

Para aplicação do método foram construídas matrizes de preferências, indicador a indicador e grupo a grupo para obter a hierarquização das empresas por meio de seus indicadores considerando o período de 2010 a 2015, ou seja, dados dos últimos seis anos. Para realizar as comparações pareadas utiliza-se a escala natural de Lootsma (1993), conforme apresentada no Quadro 1.

O método AHP é dedicado ao ambiente de tomada de decisão multicritério e muito utilizado em todo o mundo. Dessa forma, esse método foi adotado para este estudo e o resumo dos elementos de decisão dessa pesquisa apresentam-se no Quadro 3.

Quadro 3 - Elementos da aplicação no presente estudo

\begin{tabular}{|l|c|}
\hline \multicolumn{1}{|c|}{ Elemento } & Aplicação neste estudo \\
\hline Objetivo & Classificação das empresas construtoras \\
\hline Critérios de decisão & Indicadores de Liquidez e Rentabilidade \\
\hline Alternativas & 12 empresas de construção civil \\
\hline Consequência & $\begin{array}{c}\text { Empresa melhor classificada de acordo com a liquidez e } \\
\text { rentabilidade }\end{array}$ \\
\hline
\end{tabular}

Fonte: Autoria própria (2019)

\section{DESCRIÇÃO E ANÁLISE DOS DADOS}

Neste estudo foram calculados os indicadores de liquidez e de rentabilidade, conforme as fórmulas apresentadas por Gitman (2010) e Assaf Neto e Lima (2014). Após o cálculo destes indicadores, os mesmos foram submetidos à análise de componentes principais para obtenção dos scores de cada grupo de indicadores com o auxílio do SPSS versão 15.

Os scores de cada grupo de indicadores são apresentados ano a ano na Tabela 1. 
Tabela 1 - Scores dos grupos de indicadores de Liquidez e Rentabilidade

\begin{tabular}{ccccccc} 
Liquidez & 2010 & 2011 & 2012 & 2013 & 2014 & 2015 \\
Corrente & 0,1636 & 0,2082 & 0,2246 & 0,2818 & 0,2083 & 0,2735 \\
Seca & 0,1984 & 0,2150 & 0,2320 & 0,2571 & 0,1914 & 0,2348 \\
Geral & 0,1771 & 0,1960 & 0,2100 & 0,2028 & 0,1688 & 0,2112 \\
Rentabilidade & 2010 & 2011 & 2012 & 2013 & 2014 & 2015 \\
Margem LB & 0,1464 & 0,1687 & 0,1984 & 0,2124 & 0,2051 & 0,2054 \\
Margem LL & 0,0621 & 0,1333 & 0,2593 & 0,2510 & 0,2412 & 0,2642 \\
ROA & $-0,2188$ & $-0,1755$ & 0,1799 & 0,2792 & 0,2313 & 0,2246 \\
ROE & $-0,2184$ & $-0,1793$ & 0,1773 & 0,2780 & 0,2323 & 0,2206 \\
\hline
\end{tabular}

Fonte: Dados da pesquisa (2019)

Com a análise da Tabela 1, pode-se verificar no período de 2010 a 2015, que no grupo de Liquidez o maior score foi alcançado pelo indicador de Liquidez Seca nos anos de 2010 a 2012, e no período de 2013 a 2015 o indicador de Liquidez Corrente. Dentro do grupo de Rentabilidade, o índice de Margem de Lucro Bruto alcançou maior score no período de 2010 e 2011, enquanto a Margem de Lucro Líquido apresenta maior destaque no período de 2012 a 2015.

Os scores apresentados na Tabela 1 referem-se à primeira componente que permite ordenar as empresas em relação a cada grupo de indicadores por meio das ponderações obtidas.

Para a aplicação do método AHP, foi calculado anualmente em cada grupo de indicadores no período de 2010 a 2015, estabelecendo-se um ranking anual. Primeiramente, para cada grupo de indicadores foram elaboradas as matrizes de comparação utilizando os indicadores de cada empresa, onde são comparados entre si. E, cada indicador em relação a ele mesmo é de igual importância.

Para exemplificar os procedimentos de aplicação do método utilizam-se os indicadores de Liquidez Geral de 2010. Estes indicadores calculados inicialmente foram multiplicados por oito para se enquadrar à escala natural de Lootsma apresentada na Tabela 2. Conforme a Tabela 2 verificam-se os indicadores de Liquidez Geral de 2010, e seus valores convertidos para a escala natural de Lootsma (1990). 
Tabela 2 - Indicadores de Liquidez Geral de 2010 e seus valores convertidos para a escala natural de Lootsma

\begin{tabular}{cccc} 
Código & Empresas & L Geral 2010 & L Geral 2010 (multiplicado por 8) \\
E1 & Brookfield & 1,5926 & 12,7411 \\
E2 & Const A Lind & 0,4235 & 3,3881 \\
E3 & CR2 & 2,1256 & 17,0045 \\
E4 & Cyrela & 1,6361 & 13,0886 \\
E5 & Direcional & 1,9720 & 15,7761 \\
E6 & Even & 1,8540 & 14,8322 \\
E7 & Eztec & 5,2165 & 41,7317 \\
E8 & Gafisa & 1,6716 & 13,3724 \\
E9 & MRV & 1,8166 & 14,5324 \\
E10 & Rodobens & 1,4535 & 11,6281 \\
E11 & Rossi & 1,6338 & 13,0705 \\
E12 & Tecnisa & 1,6163 & 12,9302 \\
\hline
\end{tabular}

Fonte: Dados da pesquisa (2019)

Após esta conversão é realizada a comparação entre os indicadores para determinar a matriz de comparação. Para tal, utilizou-se o método das diferenças, ou seja, realiza-se uma subtração entre o indicador de cada linha com o indicador de cada coluna, o resultado, é dividido pelo menor valor entre os dois. E, cada indicador em relação a ele mesmo é de igual importância. Exemplificando:

$$
\begin{gathered}
\text { Empresa } 1 \text { (brookfield)x Empresa } 1 \text { (Brookfield) } \\
(12,7411-12,7411) / 12,7411=0 \\
\text { Empresa } 1 \text { (brookfield)x Empresa } 2 \text { (Const A Lind) } \\
(12,7411-3,3881) / 3,3881=2,7605
\end{gathered}
$$

Empresa 1 (Brookfield)x Empresa 3 (CR2)

$$
(12,7411-17,0045) / 12,7411=-0,3346
$$

Por meio deste procedimento cada empresa é comparada com as demais. Após a comparação da empresa 1 com todas as outras, compara-se a empresa 2 com as demais empresas, e assim sucessivamente até a empresa 12. Os valores obtidos compõem a matriz de comparação apresentada na Tabela 3. 
Tabela 3 - Matriz de comparação do indicador de Liquidez Geral de 2010

\begin{tabular}{|c|c|c|c|c|c|c|}
\hline & E1 & E2 & E3 & E4 & E5 & E6 \\
\hline E1 & 0,000 & $-2,760$ & 0,335 & 0,027 & 0,238 & 0,164 \\
\hline E2 & 2,760 & 0,000 & 4,019 & 2,863 & 3,656 & 3,378 \\
\hline E3 & $-0,335$ & $-4,019$ & 0,000 & $-0,299$ & $-0,078$ & $-0,146$ \\
\hline E4 & $-0,027$ & $-2,863$ & 0,299 & 0,000 & 0,205 & 0,133 \\
\hline E5 & $-0,238$ & $-3,656$ & 0,078 & $-0,205$ & 0,000 & $-0,064$ \\
\hline E6 & $-0,164$ & $-3,378$ & 0,146 & $-0,133$ & 0,064 & 0,000 \\
\hline E7 & $-2,275$ & $-11,317$ & $-1,454$ & $-2,188$ & $-1,645$ & $-1,814$ \\
\hline E8 & $-0,050$ & $-2,947$ & 0,272 & $-0,022$ & 0,180 & 0,109 \\
\hline E9 & $-0,141$ & $-3,289$ & 0,170 & $-0,110$ & 0,086 & 0,021 \\
\hline E10 & 0,096 & $-2,432$ & 0,462 & 0,126 & 0,357 & 0,276 \\
\hline E11 & $-0,026$ & $-2,858$ & 0,301 & 0,001 & 0,207 & 0,135 \\
\hline \multirow[t]{2}{*}{ E12 } & $-0,015$ & $-2,816$ & 0,315 & 0,012 & 0,220 & 0,147 \\
\hline & E7 & E8 & E9 & E10 & E11 & E12 \\
\hline E1 & 2,275 & 0,050 & 0,141 & $-0,096$ & 0,026 & 0,015 \\
\hline E2 & 11,317 & 2,947 & 3,289 & 2,432 & 2,858 & 2,816 \\
\hline E3 & 1,454 & $-0,272$ & $-0,170$ & $-0,462$ & $-0,301$ & $-0,315$ \\
\hline E4 & 2,188 & 0,022 & 0,110 & $-0,126$ & $-0,001$ & $-0,012$ \\
\hline E5 & 1,645 & $-0,180$ & $-0,086$ & $-0,357$ & $-0,207$ & $-0,220$ \\
\hline E6 & 1,814 & $-0,109$ & $-0,021$ & $-0,276$ & $-0,135$ & $-0,147$ \\
\hline E7 & 0,000 & $-2,121$ & $-1,872$ & $-2,589$ & $-2,193$ & $-2,227$ \\
\hline E8 & 2,121 & 0,000 & 0,087 & $-0,150$ & $-0,023$ & $-0,034$ \\
\hline E9 & 1,872 & $-0,087$ & 0,000 & $-0,250$ & $-0,112$ & $-0,124$ \\
\hline E10 & 2,589 & 0,150 & 0,250 & 0,000 & 0,124 & 0,112 \\
\hline E11 & 2,193 & 0,023 & 0,112 & $-0,124$ & 0,000 & $-0,011$ \\
\hline E12 & 2,227 & 0,034 & 0,124 & $-0,112$ & 0,011 & 0,000 \\
\hline
\end{tabular}

Fonte: Dados da pesquisa (2019)

Em seguida, para determinar as matrizes de preferência, consideram-se os índices $\delta \mathrm{ij} \mathrm{B}(-8,-7, \ldots, 7,8)$ do Quadro 1 para obter os valores dos $\gamma \mathrm{ij}$, ou seja, a matriz normalizada, utilizando a fórmula (GOMES; ARAYA; CARIGNANO, 2004, p. 64):

$$
\gamma_{i j}=e^{\gamma \delta_{i j}}
$$

O $\gamma$ equivale o fator de escala da escala geométrica aplicada para apontar os fatores de progressão. Lootsma (1990) indica fatores de progressão para determinado número de categorias. Assim, sugere utilizar o "fator de progressão 4, caso existam entre três e cinco categorias principais, e um fator de progressão igual a 2, caso existam entre seis e nove categorias principais. Segundo ele, será obtido um fator de escala $\gamma=0,7(\ln 2 \approx 0,7)$ " (GOMES; ARAYA; CARIGNANO, 2004, p. 64).

Baseando-se nos fatores de progressão propostos por Lootsma e considerando 12 categorias, utilizou-se o fator de progressão 1 , ou seja: $\gamma=0,35$ $(\ln 1 \approx 0,35)$. Assim, a matriz é normalizada conforme exemplo: 


$$
\begin{gathered}
\gamma_{i j}=e^{\mathbf{0 , 3 5 x 0 , 0 0}}=1,00 \\
\gamma_{i j}=e^{\mathbf{0 , 3 5 x ( - 2 , 7 6 0 )}}=0,1341
\end{gathered}
$$

Este procedimento se repete até normalizar todos os elementos da matriz. $\mathrm{Na}$ Tabela 4 apresenta-se a matriz normalizada obtida.

\begin{tabular}{|c|c|c|c|c|c|c|}
\hline & E1 & E2 & E3 & E4 & E5 & E6 \\
\hline E1 & 1,000 & 0,134 & 1,276 & 1,020 & 1,189 & 1,127 \\
\hline E2 & 7,457 & 1,000 & 18,633 & 8,035 & 14,311 & 11,685 \\
\hline E3 & 0,784 & 0,054 & 1,000 & 0,804 & 0,945 & 0,899 \\
\hline E4 & 0,980 & 0,124 & 1,243 & 1,000 & 1,161 & 1,102 \\
\hline E5 & 0,841 & 0,070 & 1,058 & 0,861 & 1,000 & 0,955 \\
\hline E6 & 0,887 & 0,086 & 1,112 & 0,908 & 1,047 & 1,000 \\
\hline E7 & 0,191 & 0,000 & 0,347 & 0,203 & 0,302 & 0,267 \\
\hline E8 & 0,965 & 0,117 & 1,219 & 0,984 & 1,140 & 1,083 \\
\hline E9 & 0,903 & 0,091 & 1,132 & 0,923 & 1,064 & 1,015 \\
\hline E10 & 1,072 & 0,170 & 1,400 & 1,096 & 1,296 & 1,222 \\
\hline E11 & 0,981 & 0,125 & 1,245 & 1,001 & 1,163 & 1,103 \\
\hline E12 & 0,989 & 0,129 & 1,258 & 1,009 & 1,174 & 1,113 \\
\hline \multirow[t]{2}{*}{ SOMA } & 17,050 & 2,100 & 30,923 & 17,844 & 25,793 & 22,570 \\
\hline & E7 & E8 & E9 & E10 & E11 & E12 \\
\hline E1 & 5,238 & 1,037 & 1,108 & 0,933 & 1,019 & 1,011 \\
\hline E2 & 1208,413 & 8,540 & 10,956 & 5,871 & 8,003 & 7,766 \\
\hline E3 & 2,882 & 0,821 & 0,884 & 0,714 & 0,803 & 0,795 \\
\hline E4 & 4,917 & 1,016 & 1,084 & 0,913 & 0,999 & 0,991 \\
\hline E5 & 3,312 & 0,877 & 0,940 & 0,771 & 0,860 & 0,852 \\
\hline E6 & 3,743 & 0,924 & 0,985 & 0,818 & 0,907 & 0,898 \\
\hline E7 & 1,000 & 0,214 & 0,256 & 0,152 & 0,203 & 0,198 \\
\hline E8 & 4,681 & 1,000 & 1,065 & 0,897 & 0,983 & 0,975 \\
\hline E9 & 3,905 & 0,939 & 1,000 & 0,834 & 0,922 & 0,914 \\
\hline E10 & 6,581 & 1,115 & 1,199 & 1,000 & 1,094 & 1,085 \\
\hline E11 & 4,933 & 1,017 & 1,085 & 0,914 & 1,000 & 0,992 \\
\hline E12 & 5,059 & 1,025 & 1,094 & 0,922 & 1,008 & 1,000 \\
\hline SOMA & 1254,664 & 18,524 & 21,655 & 14,738 & 17,802 & 17,477 \\
\hline
\end{tabular}

Tabela 4 - Matriz de Normalização

Fonte: Dados da pesquisa (2019)

Após a normalização, divide-se cada componente pela sua respectiva soma para a construção da Matriz de Prioridade Relativa, conforme apresentado na Tabela 5. 
Tabela 5 - Matriz de Prioridade Relativa

\begin{tabular}{|c|c|c|c|c|c|c|c|}
\hline & E1 & E2 & E3 & E4 & E5 & E6 & E7 \\
\hline E1 & 0,059 & 0,064 & 0,041 & 0,057 & 0,046 & 0,050 & 0,004 \\
\hline E2 & 0,437 & 0,476 & 0,603 & 0,450 & 0,555 & 0,518 & 0,963 \\
\hline E3 & 0,046 & 0,026 & 0,032 & 0,045 & 0,037 & 0,040 & 0,002 \\
\hline E4 & 0,057 & 0,059 & 0,040 & 0,056 & 0,045 & 0,049 & 0,004 \\
\hline E5 & 0,049 & 0,033 & 0,034 & 0,048 & 0,039 & 0,042 & 0,003 \\
\hline E6 & 0,052 & 0,041 & 0,036 & 0,051 & 0,041 & 0,044 & 0,003 \\
\hline E7 & 0,011 & 0,000 & 0,011 & 0,011 & 0,012 & 0,012 & 0,001 \\
\hline E8 & 0,057 & 0,056 & 0,039 & 0,055 & 0,044 & 0,048 & 0,004 \\
\hline E9 & 0,053 & 0,043 & 0,037 & 0,052 & 0,041 & 0,045 & 0,003 \\
\hline E10 & 0,063 & 0,081 & 0,045 & 0,061 & 0,050 & 0,054 & 0,005 \\
\hline E11 & 0,058 & 0,059 & 0,040 & 0,056 & 0,045 & 0,049 & 0,004 \\
\hline \multirow[t]{2}{*}{ E12 } & 0,058 & 0,061 & 0,041 & 0,057 & 0,046 & 0,049 & 0,004 \\
\hline & E8 & E9 & E10 & E11 & E12 & Prioridade & Ordem \\
\hline E1 & 0,056 & 0,051 & 0,063 & 0,057 & 0,058 & 0,044 & 3 \\
\hline E2 & 0,461 & 0,506 & 0,398 & 0,450 & 0,444 & 0,507 & 1 \\
\hline E3 & 0,044 & 0,041 & 0,048 & 0,045 & 0,045 & 0,032 & 11 \\
\hline E4 & 0,055 & 0,050 & 0,062 & 0,056 & 0,057 & 0,043 & 6 \\
\hline E5 & 0,047 & 0,043 & 0,052 & 0,048 & 0,049 & 0,035 & 10 \\
\hline E6 & 0,050 & 0,045 & 0,056 & 0,051 & 0,051 & 0,037 & 9 \\
\hline E7 & 0,012 & 0,012 & 0,010 & 0,011 & 0,011 & 0,006 & 12 \\
\hline E8 & 0,054 & 0,049 & 0,061 & 0,055 & 0,056 & 0,042 & 7 \\
\hline E9 & 0,051 & 0,046 & 0,057 & 0,052 & 0,052 & 0,038 & 8 \\
\hline E10 & 0,060 & 0,055 & 0,068 & 0,061 & 0,062 & 0,049 & 2 \\
\hline E11 & 0,055 & 0,050 & 0,062 & 0,056 & 0,057 & 0,043 & 5 \\
\hline E12 & 0,055 & 0,051 & 0,063 & 0,057 & 0,057 & 0,043 & 4 \\
\hline
\end{tabular}

Fonte: Dados da pesquisa (2019)

No passo seguinte, pode-se verificar a consistência das prioridades relativas, identificando primeiramente o vetor dos pesos das consistências.

Tabela 6 - Vetor dos Pesos das empresas

\begin{tabular}{lllllll} 
& $E 1$ & $E 2$ & $E 3$ & $E 4$ & $E 5$ & $E 6$ \\
E1 & 0,044 & 0,068 & 0,040 & 0,043 & 0,041 & 0,042 \\
E3 & 0,327 & 0,507 & 0,591 & 0,342 & 0,496 & 0,434 \\
E4 & 0,034 & 0,027 & 0,032 & 0,034 & 0,033 & 0,033 \\
E5 & 0,043 & 0,063 & 0,039 & 0,043 & 0,040 & 0,041 \\
E6 & 0,037 & 0,035 & 0,034 & 0,037 & 0,035 & 0,035 \\
E7 & 0,039 & 0,043 & 0,035 & 0,039 & 0,036 & 0,037 \\
E8 & 0,008 & 0,000 & 0,011 & 0,009 & 0,010 & 0,010 \\
E9 & 0,042 & 0,059 & 0,039 & 0,042 & 0,039 & 0,040 \\
E10 & 0,040 & 0,046 & 0,036 & 0,039 & 0,037 & 0,038 \\
E11 & 0,047 & 0,086 & 0,044 & 0,047 & 0,045 & 0,045 \\
E12 & 0,043 & 0,063 & 0,039 & 0,043 & 0,040 & 0,041 \\
\hline
\end{tabular}

Fonte: Dados da pesquisa (2019) 
Tabela 6 - Vetor dos Pesos das empresas (Continuação)

\begin{tabular}{cccccccc} 
& $E 7$ & $E 8$ & $E 9$ & $E 10$ & $E 11$ & $E 12$ & VETOR \\
E1 & 0,033 & 0,043 & 0,042 & 0,045 & 0,058 & 0,044 & 0,544 \\
E3 & 7,563 & 0,355 & 0,416 & 0,285 & 0,458 & 0,335 & 12,110 \\
E4 & 0,018 & 0,034 & 0,034 & 0,035 & 0,046 & 0,034 & 0,394 \\
E5 & 0,031 & 0,042 & 0,041 & 0,044 & 0,057 & 0,043 & 0,528 \\
E6 & 0,023 & 0,037 & 0,036 & 0,038 & 0,049 & 0,037 & 0,429 \\
E7 & 0,006 & 0,009 & 0,010 & 0,007 & 0,012 & 0,009 & 0,101 \\
E8 & 0,029 & 0,042 & 0,040 & 0,044 & 0,056 & 0,042 & 0,515 \\
E9 & 0,024 & 0,039 & 0,038 & 0,041 & 0,053 & 0,039 & 0,470 \\
E10 & 0,041 & 0,046 & 0,046 & 0,049 & 0,063 & 0,047 & 0,606 \\
E11 & 0,031 & 0,042 & 0,041 & 0,044 & 0,057 & 0,043 & 0,529 \\
E12 & 0,032 & 0,043 & 0,042 & 0,045 & 0,058 & 0,043 & 0,535 \\
\hline
\end{tabular}

Fonte: Dados da pesquisa (2019)

Continuando a verificação das consistências das prioridades relativas o passo seguinte consiste na obtenção do $\lambda$ max, apresentado na Tabela 7 , e consequentemente o índice de consistência IC (Índice de Consistência).

Tabela 7 - Vetor de consistência e $\lambda$ max

\begin{tabular}{|c|c|c|c|c|}
\hline Código & Vetor & & Prioridade & $\begin{array}{c}\text { Vetor de } \\
\text { consistência }\end{array}$ \\
\hline E1 & 0,544 & $\div$ & 0,044 & 12,402 \\
\hline E2 & 12,110 & $\div$ & 0,507 & 23,875 \\
\hline E3 & 0,394 & $\div$ & 0,032 & 12,442 \\
\hline E4 & 0,528 & $\div$ & 0,043 & 12,390 \\
\hline E5 & 0,429 & $\div$ & 0,035 & 12,392 \\
\hline E6 & 0,459 & $\div$ & 0,037 & 12,374 \\
\hline E7 & 0,101 & $\div$ & 0,006 & 16,131 \\
\hline E8 & 0,515 & $\div$ & 0,042 & 12,382 \\
\hline E9 & 0,470 & $\div$ & 0,038 & 12,372 \\
\hline E10 & 0,606 & $\div$ & 0,049 & 12,464 \\
\hline E11 & 0,529 & $\div$ & 0,057 & 9,236 \\
\hline E12 & 0,535 & $\div$ & 0,043 & 12,395 \\
\hline \multicolumn{2}{|c|}{$\lambda \max$ (média do vetor } & \multicolumn{3}{|c|}{13,4045} \\
\hline
\end{tabular}

Fonte: Dados da pesquisa (2019)

$$
\mathrm{IC}=\frac{(\lambda \max -\mathrm{n})}{\mathrm{n}-1}=\frac{(13,4045-12)}{12-1}=0,12768
$$

Finalizando a verificação das consistências dos critérios (categorias dos atrasos) utiliza-se a fórmula para calcular a taxa de consistência (CR), para assim confirmar se a avaliação do cliente quanto os critérios está ou não satisfatória.

$$
\mathrm{CR}=\frac{0,12768}{1,48}=0,08627
$$


Com isso, verifica-se que os critérios atribuídos estão consistentes e o próximo passo é a avaliação das alternativas em relação a cada critério. Da mesma forma, os procedimentos foram realizados com os indicadores de liquidez corrente, liquidez seca e liquidez geral no ano de 2010. Seguindo este procedimento obteve-se a matriz dos vetores de prioridades que é apresentada na Tabela 8.

Tabela 8 - Prioridades dos vetores de Liquidez no ano de 2010

Empresa Liquidez Corrente 2010 Liquidez Seca 2010 Liquidez Geral 2010

\begin{tabular}{clll} 
Brookfield & 0,1821 & 0,1153 & 0,0439 \\
Const A Lind & 0,8095 & 0,1718 & 0,5072 \\
CR2 & 0,0143 & 0,0640 & 0,0317 \\
Cyrela & 0,0135 & 0,0815 & 0,0426 \\
Direcional & 0,0078 & 0,0400 & 0,0346 \\
Even & 0,0101 & 0,0403 & 0,0371 \\
Eztec & 0,0051 & 0,0244 & 0,0063 \\
Gafisa & 0,0071 & 0,0312 & 0,0416 \\
MRV & 0,0108 & 0,0544 & 0,0380 \\
Rodobens & 0,0161 & 0,0744 & 0,0486 \\
Rossi & 0,0114 & 0,0560 & 0,0427 \\
Tecnisa & 0,0126 & 0,0658 & 0,0432 \\
\hline
\end{tabular}

Fonte: Dados da pesquisa (2019)

De posse da matriz de prioridades dos indicadores, o próximo passo foi elaborar a matriz de prioridade das alternativas. Para a elaboração desta matriz foram considerados os scores obtidos com a aplicação da análise de componentes principais anualmente conforme apresentados na Tabela 1. A matriz de comparação das alternativas foi elaborada a partir da comparação dos scores do grupo de indicadores de liquidez do ano de 2010, onde as alternativas são comparadas entre si. Na Tabela 9 é apresentada a matriz de comparação das alternativas.

Tabela 9 - Matriz de comparação dos scores de Liquidez geral de 2010.

\begin{tabular}{cccc} 
& Liquidez Corrente & Liquidez Seca & LiquidezGeral \\
Liquidez Corrente & 0 & 0,212602 & 0,082223 \\
Liquidez Seca & $-0,2126$ & 0 & $-0,12047$ \\
Liquidez Geral & $-0,08222$ & 0,120473 & 0 \\
\hline
\end{tabular}

Fonte: Dados da pesquisa (2019)

Utilizando a mesma técnica já descrita, a matriz de comparações foi normalizada obtendo-se a matriz apresentada na Tabela 10. 
Tabela 10 - Matriz de comparação dos scores de liquidez de 2010 normalizada

\begin{tabular}{cccc} 
& Liquidez Corrente & Liquidez Seca & Liquidez Geral \\
Liquidez Corrente & 1,000 & 1,167 & 1,062 \\
Liquidez Seca & 0,857 & 1,000 & 0,916 \\
Liquidez Geral & 0,942 & 1,092 & 1,000 \\
Soma & 2,799 & 3,259 & 2,978 \\
\hline
\end{tabular}

Fonte: Dados da pesquisa (2019)

O próximo passo foi estabelecer a posição individual de cada empresa por meio da multiplicação da matriz dos vetores de prioridade dos indicadores pela matriz do vetor de prioridade das alternativas de cada grupo de indicadores, conforme apresentado na Tabela 11.

Tabela 11 - Matriz de prioridades de Liquidez

\begin{tabular}{cccccc} 
& Liquidez & Liquidez Seca & Liquidez Geral & Prioridade & Ordem \\
Liquidez Corrente & 0,3573 & 0,3582 & 0,3565 & 0,3574 & 1 \\
Liquidez Seca & 0,3061 & 0,3068 & 0,3076 & 0,3069 & 3 \\
Liquidez Geral & 0,3366 & 0,3350 & 0,3358 & 0,3358 & 2 \\
\hline
\end{tabular}

Fonte: Dados da pesquisa (2019)

O próximo passo foi estabelecer a posição individual de cada empresa por meio da multiplicação da matriz dos vetores de prioridade dos indicadores pela matriz do vetor de prioridade das alternativas de cada grupo de indicadores. No caso dos indicadores de liquidez é efetuada a seguinte multiplicação.

Figura 1 - Multiplicação das matrizes

$$
\left[\begin{array}{lll}
0,1821 & 0,1153 & 0,0439 \\
0,8095 & 0,1718 & 0,5072 \\
0,0143 & 0,0640 & 0,0317 \\
0,0135 & 0,0815 & 0,0426 \\
0,0078 & 0,0400 & 0,0346 \\
0,0101 & 0,0403 & 0,0371 \\
0,0051 & 0,0244 & 0,0063 \\
0,0071 & 0,0312 & 0,0416 \\
0,0108 & 0,0544 & 0,0380 \\
0,0161 & 0,0744 & 0,0486 \\
0,0114 & 0,0560 & 0,0427 \\
0,0126 & 0,0658 & 0,0432
\end{array}\right] \times\left[\begin{array}{l}
0,3574 \\
0,3069 \\
0,3358
\end{array}\right]=\left[\begin{array}{l}
0,1152 \\
0,5124 \\
0,0354 \\
0,0441 \\
0,0267 \\
0,0284 \\
0,0114 \\
0,0261 \\
0,0333 \\
0,0449 \\
0,0356 \\
0,0392
\end{array}\right.
$$

Fonte: Dados da pesquisa (2019)

Realizada a multiplicação dessas matrizes obtém-se a ordenação das empresas demonstrando a empresa que obteve o melhor desempenho em relação aos indicadores de liquidez de 2010 a 2015. Os rankings referentes aos indicadores de liquidez são apresentados no Quadro 4. 
Quadro 4-Ranking das empresas em relação a Liquidez de 2010 a 2015

\begin{tabular}{|c|c|c|c|c|c|c|}
\hline & $\mathbf{2 0 1 0}$ & $\mathbf{2 0 1 1}$ & $\mathbf{2 0 1 2}$ & $\mathbf{2 0 1 3}$ & $\mathbf{2 0 1 4}$ & $\mathbf{2 0 1 5}$ \\
\hline 1 & Const A Lind & Even & Const A Lind & $\begin{array}{c}\text { Const A } \\
\text { Lind }\end{array}$ & Rossi & Rossi \\
\hline 2 & Brookfield & Const A Lind & Brookfield & Rossi & Brookfield & Brookfield \\
\hline 3 & Rodobens & Gafisa & Rossi & Brookfield & Tecnisa & Tecnisa \\
\hline 4 & Cyrela & Brookfield & MRV & Tecnisa & $\begin{array}{c}\text { Const A } \\
\text { Lind }\end{array}$ & Gafisa \\
\hline 5 & Tecnisa & Cyrela & Tecnisa & Gafisa & Gafisa & MRV \\
\hline 6 & Rossi & MRV & CR2 & MRV & MRV & Const A Lind \\
\hline 7 & CR2 & Rossi & Cyrela & Cyrela & Rodobens & Rodobens \\
\hline 8 & MRV & CR2 & Gafisa & Rodobens & Cyrela & Cyrela \\
\hline 9 & Even & Tecnisa & Rodobens & Direcional & Direcional & Even \\
\hline 10 & Direcional & Direcional & Direcional & Even & Even & Direcional \\
\hline 11 & Gafisa & Rodobens & Even & Eztec & Eztec & Eztec \\
\hline 12 & Eztec & Eztec & Eztec & CR2 & CR2 & CR2 \\
\hline
\end{tabular}

Fonte: Dados da pesquisa (2019)

Para a obtenção dos rankings dos indicadores de rentabilidade são realizados os mesmos procedimentos apresentados. Seguindo o mesmo raciocínio obtêm-se os rankings anualmente.

O desempenho em relação aos indicadores de rentabilidade também foi analisado o ranking resultante da aplicação do método AHP é apresentada no Quadro 5.

Quadro 5 - Ranking das empresas em relação a Rentabilidade

\begin{tabular}{|c|c|c|c|c|c|c|}
\hline & $\mathbf{2 0 1 0}$ & $\mathbf{2 0 1 1}$ & $\mathbf{2 0 1 2}$ & $\mathbf{2 0 1 3}$ & $\mathbf{2 0 1 4}$ & $\mathbf{2 0 1 5}$ \\
\hline $\mathbf{1}$ & Rossi & Rossi & Brookfield & Rossi & Tecnisa & Rodobens \\
\hline 2 & Brookfield & CR2 & MRV & MRV & Direcional & Even \\
\hline 3 & Gafisa & Gafisa & Rodobens & Tecnisa & Even & Gafisa \\
\hline 4 & CR2 & Rodobens & Even & Cyrela & Rodobens & Direcional \\
\hline 5 & Even & Tecnisa & Direcional & Even & Cyrela & MRV \\
\hline 6 & Direcional & Const A Lind & Cyrela & CR2 & MRV & Rossi \\
\hline 7 & MRV & Brookfield & Const A Lind & Eztec & Brookfield & Eztec \\
\hline 8 & Tecnisa & Cyrela & Eztec & Direcional & Rossi & Tecnisa \\
\hline 9 & Cyrela & Even & Tecnisa & Gafisa & Const A Lind & Const A Lind \\
\hline 10 & Rodobens & Direcional & Rossi & Rodobens & Eztec & CR2 \\
\hline 11 & Eztec & MRV & CR2 & Const A Lind & CR2 & Eztec \\
\hline 12 & Const A Lind & Eztec & Gafisa & Brookfield & Gafisa & Brookfield \\
\hline
\end{tabular}

Fonte: Dados da pesquisa (2019)

Com os rankings parciais de cada ano e de cada grupo de indicadores, estabeleceu-se um ranking geral em relação a cada grupo e o período analisado. Para estabelecer esse ranking geral a partir dos rankings parciais, somou-se a 
pontuação de cada empresa ao longo dos anos analisados em cada grupo de indicadores. No Quadro 6 são apresentados os rankings obtidos mediante esse procedimento.

Quadro 6 - Ranking geral das empresas em relação à liquidez e rentabilidade de 2010 a 2015

\begin{tabular}{|c|c|c|}
\hline Ranking & Liquidez & Rentabilidade \\
\hline 1 & Const A Lind & Rossi \\
\hline 2 & Rossi & Rodobens \\
\hline 3 & Brookfield & Gafisa \\
\hline 4 & Tecnisa & Direcional \\
\hline 5 & MRV & Brookfield \\
\hline 6 & Gafisa & MRV \\
\hline 7 & Rodobens & CR2 \\
\hline 8 & Cyrela & Tecnisa \\
\hline 9 & Even & Const A Lind \\
\hline 10 & CR2 & Eztec \\
\hline 11 & Direcional & \\
\hline 12 & Eztec & \\
\hline
\end{tabular}

Fonte: Dados da pesquisa (2019)

Verifica-se de acordo com o Quadro 6, que a empresa Rossi apresenta uma melhor posição dentre as demais, visto que ocupa a segunda e primeira colocação nos indicadores de liquidez e rentabilidade, respectivamente. A empresa Eztec apresentou a pior colocação tanto em liquidez, quanto em rentabilidade. Já a Construtora Adolph Lindenberg, primeira colocada nos indicadores de liquidez, encontra-se na 11a colocação destacando um maior conflito entre os indicadores. Isto corrobora com Assaf Neto e Lima (2014), que esclarece que a empresa pode possuir boa capacidade de liquidar suas obrigações, contudo não necessariamente apresentará o mesmo potencial de rentabilidade.

\section{CONSIDERAÇÕES FINAIS}

A avaliação de desempenho organizacional é importante para verificar se os objetivos estabelecidos pela empresa estão sendo alcançados, ajudando ainda na melhor aplicação dos recursos. Para mensuração do desempenho, procura-se medir as consequências financeiras e econômicas das decisões de gestões passadas.

Para efetuar a mensuração do desempenho, utiliza-se a apuração de indicadores para fornecer uma ampla visão da situação econômica, financeira e patrimonial da empresa. Os principais aspectos revelados pelos índices econômico-financeiros são divididos em três grupos, sendo: Liquidez e Estrutura de Capital - evidenciam aspectos financeiros; Rentabilidade - evidencia aspectos econômicos; e Valor de Mercado - evidencia os aspectos de risco e rentabilidade.

Este estudo objetivou definir por meio dos indicadores de liquidez e rentabilidade, um ranking das empresas do setor de construção civil listadas na 
BMF\&Bovespa indicadas como maiores empresas pelo ICT em 2014 e 2015, com a utilização do método AHP Multiplicativo. Para atender ao objetivo, foi utilizada uma metodologia descritiva, realizada por meio de análise documental, com abordagem quantitativa. Foram utilizados os indicadores extraídos das demonstrações contábeis compreendidas entre 2010 a 2015 e, sobre eles, aplicado o método AHP Multiplicativo.

Em relação aos indicadores de liquidez, a empresa Construtora Adolpho Lindenberg destaca-se nos anos de 2010, 2012 e 2013. Em 2011 a empresa Even manteve a primeira posição e a Construtora Adolpho Lindenberg ocupa a segunda posição, contudo em 2014 e 2015 a empresa Rossi apresenta o melhor resultado seguida pela empresa Brookfield na segunda colocação em ambos exercícios.

Inversamente, em destaque pela última posição encontra-se a empresa Eztec nos anos de 2010, 2011, 2012 e a empresa CR2 nos anos de 2013, 2014 e 2015.

Nos indicadores de rentabilidade se destaca em 2010 a empresa Rossi, com a segunda colocação a empresa Brookfield. Em 2012 novamente se destaca a empresa Rossi, seguida pela empresa CR2. Em 2012, Brookfield e MRV ocupam a primeira e segunda colocação respectivamente. Já em 2013, Rossi e MRV apresentam os melhores resultados. A empresa Tecnisa e Rodobens apresentam os melhores resultados nos anos de 2014 e 2015 respectivamente.

Desta forma, foi possível estabelecer um ranking geral a partir dos rankings parciais, obtendo-se o posicionamento geral de cada empresa em relação a cada grupo de indicadores. As empresas Construtoras Adolpho Lindenberg e a Rossi apresentam os melhores resultados nos indicadores de liquidez, enquanto as empresas Rossi e Rodobens se destacam nos indicadores de rentabilidade.

Destaca-se o desempenho da empresa Ezetec que em ambos indicadores apresentou a última colocação, e a Construtora Adolpho Lindenberg que mesmo com a primeira colocação nos indicadores de liquidez, apresenta a 11a colocação nos indicadores de rentabilidade.

$\mathrm{Na}$ teoria, verificou-se que a empresa pode possuir boa capacidade de liquidar suas obrigações, contudo não necessariamente apresentará o mesmo potencial de rentabilidade, ou seja, a empresa pode não usufruir de bom desempenho econômico e vice-versa.

Apesar de este trabalho ter sido focado em apenas em dois indicadores, liquidez e rentabilidade, entende-se que o Método AHP Multiplicativo possa ser aplicado aos demais indicadores assim como em diversas situações do ambiente de decisões complexas e servir de apoio as revistas especializadas para a construção de novos rankings que considerem o desempenho econômicofinanceiro das empresas construtoras, como também a investidores e demais stakerholders do setor.

Verifica-se a aplicabilidade eficiente do Método AHP Multiplicativo em que ocorra o processo de decisão entre uma série de alternativas conflitantes e concorrentes, tais como seleção de terrenos para a construção de empreendimentos, seleção de fornecedores, seleção de investimentos, entre outros. 
Para futuros trabalhos, sugere-se: i) desenvolver estudos com outros métodos multicritérios para realizar comparações; ii) verificar o impacto da situação política e econômica no desempenho do setor; iii) ampliar a pesquisa para outros setores da economia. 


\title{
A Multi-criteria Analysis of the Liquidity and Profitability Indicators of Brazilian Civil Construction Companies
}

\begin{abstract}
The research aims to define the ranking of companies in the civil construction sector listed on the BM \& FBovespa based on liquidity and profitability indicators using the Analytic Hierarchy Process, AHP Multiplicative. For the analysis, the data of the financial statements: Balance Sheets and Statement of Income (DRE), extracted from the website of the Brazilian Securities and Exchange Commission (CVM) and analyzed using the Multiplicative AHP Method were used to establish the annual and general rankings . It was verified that in the liquidity indicators, the following companies stand out: Adolpho Lindenberg (in 2010, 2012 and 2013), Rossi (2014 and 2015) and Even (in 2011). Already in the profitability indicators, the companies Rossi (in 2010, 2011 and 2013), Brookfield (in 2012), Tecnisa (in 2014) and Rodobens (in 2015) stand out. With the results, a general ranking was elaborated in relation to the analyzed period, highlighting firstly Adolpho Lindenberg and secondly Rossi. The results corroborate Assaf Neto and Lima (2014), which clarifies that the company may have a good ability to settle its obligations, but it does not necessarily have the same potential for profitability, i.e., the company may not enjoy a good economic result.
\end{abstract}

KEYWORDS: Analysis of indicators. Liquidity. Profitability. Multiplicative AHP. 
ALTAMIRANO CORRO, A.; ZAMORA ANTUÑANO, M. A.; GÓMEZ MELÉNDEZ, D. J.; PENICHE VERA, R. R. Medición de la Eficiencia Institucional en la Educacion Pública a través del Proceso de Jerarquía Analítica. El Caso de La Universidad Autónoma de Querétaro, México. Interciencia, Noviembre-Sin mes, 779-783, 2015.

ASSAF NETO, Alexandre; LIMA, Fabiano Guasti. Curso de Administração financeira. 3a. ed. São Paulo: Atlas, 2014.

GIL, Antônio Carlos. Como elaborar projetos de pesquisa. 6ạ ed. São Paulo: Atlas, 2017.

GITMAN, Lawrence J. Princípios de administração financeira. 12a ed. São Paulo: Pearson Pratice Hall, 2010.

GOMES, L. F. A. M., ARAYA, M. C. G., CARIGNANO, C. Tomada de decisões em cenários complexos. São Paulo: Pioneira Thompson Learning, 2004;

HERNÁNDEZ, C. T.; MARINS, F. A. S.; CASTRO, R. C. Modelo de Gerenciamento da Logística Reversa. Revista Gestão e Produção, São Carlos, v. 19, n. 3, p. 445-456, 2012. crossref

IUDÍCIBUS, Sérgio de. Análise de Balanços. 11ạ. ed. São Paulo: Atlas, 2017.

LOOTSMA, F. A. Scale sensitivity in the multiplicative AHP and SMART. Journal of Multi-Criteria Decision Analysis, 2(2), 87-110, 1993. crossref

MATTANA, A. J.; MEDEIROS, M. H. F. DE; SILVA, N. G. DA; COSTA, M. DO R. DE M. M. da. Análise hierárquica para escolha entre agregado natural e areia de britagem de rocha para confecção de argamassas de revestimento. Ambiente Construído, Porto Alegre, v. 12, n. 4, p. 63-79, out./dez. 2012. crossref

MENEZES, P. C. F. de. Sistemas Lógicos Paraconsistentes Aplicados aos Modelos Hierárquicos Para Tomadas de Decisão: Estudo Realizados em Gestão de Projetos. 2015. 102 f. Dissertação (Mestrado em Engenharia Mecânica) -Programa de Pós-Graduação em Engenharia Mecânica, Universidade Santa Cecília, Santos, 2015.

PEREIRA, E.; MEDEIROS, M. H. F.; LEVY, S. M. Durabilidade de Concretos Com Agregados Reciclados: uma aplicação de análise hierárquica. Ambiente Construído, Porto Alegre, v. 12, n. 3, p. 125-134, jul./set. 2012. crossref 
SAATY, T. L. Método de análise hierárquica. São Paulo: McGraw-Hill; Makron, 1991.

SAATY, T. L. Decision making with the analytic hierarchy process. Int. J. Services Sciences, Vol. 1, No. 1, 2008. crossref

SAATY, T. L. The Modern Science of Multicriteria Decision Making and Its Practical Applications: The AHP/ANP. Approach Operations Research, Vol. 61, No. 5, September-October, pp. 1101-1118, 2013. crossref

SILVA, A. A. da. Estrutura, Análise e Interpretação das Demonstrações Contábeis. 4 ed. São Paulo: Atlas, 2014.

SHIMIZU, T. Decisão nas Organizações. 2ª ed. São Paulo: Atlas, 2006.

SPSS, Statistical Package for the Social Sciences, Level M Ver. 15.0, Chicago IL

SIMÃO, A.S.. et al. Uma análise multicritério dos indicadores de Liquidez e Rentabilidade de Empresas Brasileiras da Construção Civil. R. Gest. Industr., Ponta Grossa, v. 15, n. 3, p. 17-42, Jul../Set. 2019.

Disponível em: <https://periodicos.utfpr.edu.br/revistagi> . Acesso em:

Correspondência:

Alessandra dos Santos Simão

Universidade Federal Fluminense (UFF), Niterói, Rio de Janeiro, Brasil.

Direito autoral: Este artigo está licenciado sob os termos da Licença Creative Commons-Atribuição 4.0 Internacional.

\section{(c) (i)}




\section{APÊNDICE}

Você está participando de uma pesquisa de campo na mensuração sobre a importância da utilização de indicadores para avaliação do desempenho empresarial, tendo apenas o objetivo de validação dos procedimentos científicos de nossa pesquisa. Desde já, agradecemos sua participação.

1) Considerando os grupos de indicadores, assinale um número de 1 a 5 utilizando a escala abaixo, para indicar quão importante você os considera para analisar o desempenho empresarial

\begin{tabular}{|l|l|l|l|l|}
\hline $\begin{array}{l}\text { Nada importante } \\
\text { Naco }\end{array}$ & $\begin{array}{l}2- \\
\text { Pouco } \\
\text { importante }\end{array}$ & $\begin{array}{l}\text { 3-Mediamente } \\
\text { importante }\end{array}$ & $\begin{array}{l}4- \\
\text { Muito } \\
\text { importante }\end{array}$ & $\begin{array}{l}5- \\
\text { Totalmente } \\
\text { importante }\end{array}$ \\
\hline
\end{tabular}

Indicadores de Estrutura de capital ( ) Indicadores de Liquidez ( ) Indicadores de Rentabilidade ( )

Indicadores de Atividade ( ) Indicadores de Valor de Mercado ( )

2) Considerando os diferentes indicadores, assinale um número de 1 a 5 utilizando a escala abaixo, para indicar quão importante você os considera para analisar o desempenho empresarial

\begin{tabular}{|l|l|l|l|l|}
\hline $\begin{array}{l}\text { N- } \\
\text { Nada importante }\end{array}$ & $\begin{array}{l}2- \\
\text { Pouco } \\
\text { importante }\end{array}$ & $\begin{array}{l}\text { 3-Mediamente } \\
\text { importante }\end{array}$ & $\begin{array}{l}4- \\
\text { Muito } \\
\text { importante }\end{array}$ & $\begin{array}{l}\text { 5- } \\
\text { Totalmente } \\
\text { importante }\end{array}$ \\
\hline
\end{tabular}

\begin{tabular}{|c|c|c|c|c|}
\hline & Indicador & Fórmula & Interpretação & Pontos \\
\hline \multirow[t]{4}{*}{$\begin{array}{l}\text { ESTRUTURA DE } \\
\text { CAPITAL }\end{array}$} & $\begin{array}{l}\text { Participação de } \\
\text { Capitais de } \\
\text { Terceiros }\end{array}$ & $\begin{array}{l}\frac{\text { Capitais de }}{\text { Terceiros } \times 100} \\
\text { Patrimônio } \\
\text { Líquido }\end{array}$ & $\begin{array}{l}\text { Quanto a } \\
\text { empresa } \\
\text { tomou de } \\
\text { capitais de } \\
\text { terceiros para } \\
\text { cada } \$ 100 \text { de } \\
\text { capital próprio }\end{array}$ & \\
\hline & $\begin{array}{l}\text { Composição do } \\
\text { Endividamento }\end{array}$ & $\begin{array}{l}\frac{\text { Passivo }}{\text { Circulante }} \\
\text { x100 } \\
\text { Capitais de } \\
\text { Terceiros }\end{array}$ & $\begin{array}{l}\text { Qual } \\
\text { percentual de } \\
\text { obrigações a } \\
\text { curto prazo em } \\
\text { relação ao } \\
\text { total das } \\
\text { obrigações } \\
\end{array}$ & \\
\hline & $\begin{array}{l}\text { Imobilização } \\
\text { do Patrimônio } \\
\text { Líquido }\end{array}$ & $\begin{array}{l}\frac{\text { Ativo Não }}{\text { Circulante }} \times 100 \\
\text { Patrimônio } \\
\text { Líquido }\end{array}$ & $\begin{array}{l}\text { Valor em R\$ } \\
\text { que a empresa } \\
\text { aplicou no } \\
\text { Imobilizado } \\
\text { para cada } \$ 100 \\
\text { de Patr. } \\
\text { Líquido }\end{array}$ & \\
\hline & $\begin{array}{l}\text { Imobilização } \\
\text { dos Recur. não } \\
\text { Correntes }\end{array}$ & $\begin{array}{l}\frac{\text { Imobilizado }}{\text { x100 }} \\
\text { Passivo não } \\
\text { Circ+ Pat } \\
\text { Líquido }\end{array}$ & $\begin{array}{l}\text { Valor em R\$ } \\
\text { que a empresa } \\
\text { aplicou no } \\
\text { Imobilizado } \\
\text { para cada \$100 } \\
\text { de Patr. } \\
\text { Líquido + } \\
\text { Passivo Não } \\
\text { Circulante }\end{array}$ & \\
\hline
\end{tabular}




\begin{tabular}{|c|c|c|c|c|}
\hline & Indicador & Fórmula & Interpretação & Pontos \\
\hline \multirow[t]{4}{*}{ LIQUIDEZ } & $\begin{array}{l}\text { Liquidez } \\
\text { Corrente }\end{array}$ & $\frac{\text { Ativo Circulante }}{\text { Passivo Circulante }}$ & $\begin{array}{l}\text { Quanto a empresa } \\
\text { possui de Ativo } \\
\text { Circulante para } \\
\text { cada } \$ 1 \text { de Passivo } \\
\text { Circulante }\end{array}$ & \\
\hline & Liquidez Seca & $\begin{array}{l}\text { Ativo Circulante }(-) \\
\frac{\text { Estoques }}{\text { Passivo Circulante }}\end{array}$ & $\begin{array}{l}\text { Quanto a empresa } \\
\text { possui de Ativo } \\
\text { Líquido (exceto } \\
\text { Estoque) p/ cada } \\
\text { \$1 de Passivo } \\
\text { Circulante }\end{array}$ & \\
\hline & $\begin{array}{l}\text { Liquidez } \\
\text { Imediata }\end{array}$ & $\begin{array}{l}\text { Disponibilidades } \\
\text { Passivo Circulante }\end{array}$ & $\begin{array}{l}\text { Quanto a empresa } \\
\text { possui de possuiu } \\
\text { de dinheiro em } \\
\text { caixa, bancos e em } \\
\text { aplicações de } \\
\text { liquidez imediata } \\
\text { p/ cada \$1 de } \\
\text { Passivo Circulante }\end{array}$ & \\
\hline & Liquidez Geral & $\begin{array}{l}\text { AtivoCirc+Ativo n Circ } \\
\text { PassivoCirc+Passivo } n \\
\text { Circ }\end{array}$ & $\begin{array}{l}\text { Quanto a empresa } \\
\text { possui de Ativo } \\
\text { Circulante + Ativo } \\
\text { Não Circulante p/ } \\
\text { cada \$1 de Dívida } \\
\text { Total }\end{array}$ & \\
\hline
\end{tabular}

\begin{tabular}{|c|c|c|c|c|}
\hline & Indicador & Fórmula & Interpretação & Pontos \\
\hline \multirow[t]{4}{*}{ Rentabilidade } & Giro do Ativo & $\begin{array}{l}\text { Vendas Líquidasx } 1 \\
\text { Ativo Total }\end{array}$ & $\begin{array}{l}\text { Quanto a } \\
\text { empresa } \\
\text { vendeu para } \\
\text { cada } \$ 1 \text { de } \\
\text { Ativo Total }\end{array}$ & \\
\hline & $\begin{array}{l}\text { Margem } \\
\text { Líquida }\end{array}$ & $\begin{array}{l}\text { Lucro Líquido } \times 100 \\
\text { Vendas Líquidas }\end{array}$ & $\begin{array}{l}\text { Quanto a } \\
\text { empresa } \\
\text { obtém de } \\
\text { lucro para } \\
\text { cada } \$ 100 \\
\text { vendidos }\end{array}$ & \\
\hline & $\begin{array}{l}\text { Margem } \\
\text { Líquida }\end{array}$ & $\frac{\text { Lucro Líquido }}{\text { Ativo Total }} \times 100$ & $\begin{array}{l}\text { Quanto a } \\
\text { empresa } \\
\text { obtém de } \\
\text { lucro para } \\
\text { cada \$100 de } \\
\text { Investimento } \\
\text { total }\end{array}$ & \\
\hline & $\begin{array}{l}\text { Rentabilidade } \\
\text { do Pat. Líquido }\end{array}$ & $\begin{array}{l}\text { Lucro Líquido x100 } \\
\text { Patr.Líquido Médio }\end{array}$ & $\begin{array}{l}\text { Quanto a } \\
\text { empresa } \\
\text { obtém de } \\
\text { lucro para } \\
\text { cada } \$ 100 \text { de } \\
\text { capital próprio } \\
\text { investido } \\
\text { (médio) }\end{array}$ & \\
\hline
\end{tabular}




\begin{tabular}{|c|c|c|c|c|}
\hline & Indicador & Fórmula & Interpretação & Pontos \\
\hline \multirow[t]{4}{*}{ Atividade } & $\begin{array}{l}\text { PM } \\
\text { Recebimento } \\
\text { Vendas }\end{array}$ & $\frac{\text { Clientes }}{\text { Vendas }} \times 360$ & $\begin{array}{l}\text { Prazo (em dias) que a } \\
\text { empresa consegue } \\
\text { receber suas vendas }\end{array}$ & \\
\hline & $\begin{array}{l}\text { PM Pagamento } \\
\text { de Compras }\end{array}$ & $\frac{\text { Fornecedores }}{\text { Compras }} \times 360$ & $\begin{array}{l}\text { Prazo (em dias) que a } \\
\text { empresa consegue } \\
\text { pagar suas compras }\end{array}$ & \\
\hline & $\begin{array}{l}\text { PM Rotação de } \\
\text { Estoques }\end{array}$ & $\frac{\text { Estoques }}{\mathrm{CMV}} \times 360$ & $\begin{array}{l}\text { Prazo (em dias) que a } \\
\text { empresa leva para } \\
\text { renovar o estoque }\end{array}$ & \\
\hline & $\begin{array}{l}\text { Ciclo } \\
\text { Operacional }\end{array}$ & $\begin{array}{l}\text { (PM Rotação de } \\
\text { Estoques + PM } \\
\text { Recebimento de } \\
\text { Vendas) - PM } \\
\text { Pagamento de } \\
\text { Compras }\end{array}$ & $\begin{array}{l}\text { Compreende o } \\
\text { período entre a data } \\
\text { da compra até o } \\
\text { recebimento de } \\
\text { cliente. }\end{array}$ & \\
\hline
\end{tabular}

\begin{tabular}{|c|c|c|c|c|}
\hline & Indicador & Fórmula & Interpretação & Pontos \\
\hline \multirow[t]{3}{*}{$\begin{array}{l}\text { VALOR DE } \\
\text { MERCADO }\end{array}$} & $\begin{array}{l}\text { Lucro por } \\
\text { Ação (LPA) }\end{array}$ & $\frac{\text { Lucro Líquido }}{\mathrm{N}^{\circ} \text { de ações emitidas }}$ & $\begin{array}{l}\text { É o montante obtido } \\
\text { durante o período } \\
\text { por cada ação } \\
\text { ordinária }\end{array}$ & \\
\hline & $\begin{array}{l}\text { Índice } \\
\text { Preço/Lucro }\end{array}$ & $\frac{\text { Preço de Mercado por }}{\frac{\text { ação ordinária / Lucro por }}{\text { ação (LPA) }}}$ & $\begin{array}{l}\text { Mede o valor que os } \\
\text { investidores estão } \\
\text { dispostos a pagar por } \\
\mathrm{R} \$ 1 \text { de lucro. }\end{array}$ & \\
\hline & $\begin{array}{l}\text { Preço/Valor } \\
\text { Patrimonial }\end{array}$ & $\begin{array}{l}\text { Valor de Mercado por } \\
\text { ação ordinária / Valor } \\
\text { Contábil por ação } \\
\text { ordinária }\end{array}$ & $\begin{array}{l}\text { Fornece uma } \\
\text { avaliação de como os } \\
\text { investidores encaram } \\
\text { o desempenho da } \\
\text { empresa }\end{array}$ & \\
\hline
\end{tabular}

